\title{
Decreased ARID1A Expression Associates with Tumor Progression and Adverse Prognosis in Renal Cell Carcinoma
}

\author{
Keerakarn Somsuan \\ Mae Fah Luang University \\ Ratirath Samon \\ Sawanpracharak Hospital \\ Paween Tangjitpisud \\ Mae Fah Luang University \\ Siripat Aluksanasuwan \\ Mae Fah Luang University \\ Yupa Srithongchai \\ Naresuan University \\ Visith Thongboonkerd \\ Siriraj Hospital \\ NATTHIYA SAKULSAK ( $\nabla$ natthiyak@nu.ac.th ) \\ Naresuan University
}

\section{Research Article}

Keywords: ARID1A, renal cell carcinoma, pathological outcomes, cancer prognostic marker

Posted Date: November 17th, 2021

DOI: https://doi.org/10.21203/rs.3.rs-1034681/v1

License: (9) This work is licensed under a Creative Commons Attribution 4.0 International License. Read Full License 


\section{Abstract}

In this study, we aimed to evaluate association of ARID1A (AT-rich interacting domain-containing protein $1 A$ ) mutation and protein expression with clinicopathology and prognosis of renal cell carcinoma (RCC). Genomic Data Commons (GDC) showed ARID1A was one of the top-ten mutated genes found in kidney cancers and its mutations were found along its sequence. Interestingly, patients with ARID1A mutations had significantly lower survival rate $(38 \% ; n=68)$ comparing to the non-mutated cases $(58 \% ; n=192)$. The results from OSkirc web tool revealed that patients with low expression of $A R I D 1 A$ had significantly shorter overall survival and disease specific survival than those with high ARID1A expression.

Immunohistochemistry revealed markedly decreased ARID1A expression in the RCC tissues $(n=26)$, particularly in clear cell RCC (ccRCC) and chromophobe RCC (chRCC). Negative to weak ARID1A expression was significantly associated with ccRCC (grade II) and chRCC subtypes, presence of comorbidity, and low eGFR levels. Finally, ARID1A protein was undetectable in 3/11 cases with ccRCC (grade II) and 2/6 chRCC cases, all of which had metastasis 1-50 months after surgical removal. In conclusion, decreased ARID1A expression is associated with the poor prognosis and metastasis of RCC and thus may serve as the prognostic marker of RCC, particularly $\mathrm{CCRCC}$ and chRCC subtypes.

\section{Introduction}

ARID1A encoding AT-rich interacting domain-containing protein $1 \mathrm{~A}$ is one of the tumor suppressor genes, mutations of which have been found in various cancers, particularly ovarian clear cell carcinoma (57\%), gastric cancer (29\%), and bladder transitional cell carcinoma (13\%) [1]. Several previous studies have investigated pathogenic mechanisms of defective ARID1A in the development and progression of cancers. One of these studies has shown that silencing of $A R I D 1 A$ in gastric cancer cells inhibited transcription of $\mathrm{CDH}-1$ (encoding E-cadherin) and subsequently induced accumulation of $\beta$-catenin in the nucleus [2]. Such accumulation could enhance the Wnt/ $\beta$-catenin pathway that regulates migration and invasion of gastric cancer cells [2]. A recent study has also demonstrated that inactivation of $A R I D 1 A$ in mice could facilitate colon tumorigenesis via defective APC/ $\beta$-catenin and SWI/SNF pathways [3]. Decrease of ARID1A mRNA expression has been shown to be involved with promoter hypermethylation in breast invasive ductal carcinoma and squamous cell carcinoma [4, 5]. Interestingly, a decrease (approximately 30-40 \%) in ARID1A transcriptional activity has been observed in renal cell carcinoma (RCC) [6]. Moreover, the loss of ARID1A protein expression in tumor tissues has been observed in approximately $67 \%$ of patients with clear cell RCC (cCRCC) [7]. In addition, the loss of ARID1A protein expression in tumor tissues has been found to be associated with poor clinicopathological outcome of ccRCC patients, including large tumor size $(>7 \mathrm{~cm})$, high Fuhrman nuclear grade (III/IV), high pTNM stage (III) and relatively short progression-free interval $[7,8]$. These studies have implied that the loss of ARID1A protein expression in tumor tissues might serve as a prognostic marker in RCC.

Nevertheless, the relevance of $A R I D 1 A$ down-regulation in RCC has remained under-investigated. We thus evaluated the association of $A R I D 1 A$ gene mutation and ARID1A protein expression with clinicopathology 
and prognosis in various types of RCC, including ccRCC (grade I), ccRCC (grade II), chromophobe RCC (chRCC), papillary RCC (pRCC), and sarcomatoid RCC (sRCC).

\section{Materials \& Methods}

\section{Bioinformatics analysis}

The mutated genes commonly found in kidney cancers were analyzed through the Genomic Data Commons (GDC) database (http://portal.gdc.cancer.gov/). All of The Cancer Genome Atlas (TCGA) projects related to kidney cancers, including TCGA-KIRC (kidney renal clear cell carcinoma), TCGA-KIRP (kidney renal papillary cell carcinoma), TCGA-KICH (kidney chromophobe) and TCGA-SARC (sarcoma), which recruited 943 patients (accessed on October 11, 2019), were analyzed. From this information the number of patients affected by each of the top-20 mutated genes was recorded. Additionally, the profile of ARID1A mutations along the protein-coding regions and their frequency were investigated. Moreover, Kaplan-Meier curve and the log-rank test were conducted to estimate the overall survival (OS) of patients with mutated ARID1A compared to those without ARID1A mutations. In addition, the Online consensus Survival analysis for kidney renal clear cell carcinoma (KIRC), OSkirc web tool (http://bioinfo.henu.edu.cn/KIRC/KIRCList.jsp) was used to verify the prognostic outcomes of $A R I D 1 A$ gene in patients with $\mathrm{KIRC}$, as recorded in a previous study [9]. The expression profiling of ARID1A gene and clinical follow-up information of patients were accessed from The Cancer Genome Atlas (TCGA) and the Gene Expression Omnibus (GEO) databases. The Kaplan-Meier survival plots of overall survival (OS) and disease specific survival (DSS) with hazard ratio (HR) and log-rank $p$-value were also examined.

\section{RCC patients and tissues}

RCC patients admitted to Sawanpracharak Hospital, Nakhon Sawan, Thailand for nephrectomy during 2013-2017 were recruited, whereas those with hereditary RCC syndrome were excluded. The participants were asked to give an informed consent. All study procedures were approved by the Human Ethic Review Board of Sawanpracharak Hospital and by Naresuan University Ethical Committee for Human Research (NU-IRB) and was conducted in accordance with the Declaration of Helsinki Principles. Clinical data, i.e., age, gender, tumor laterality, pathological subtype and staging (TNM and AJCC systems), tumor mass diameter, tumor invasion, metastasis, recurrence, and other complications, were extracted and analyzed. Their paired renal tissues, including cancer and adjacent non-cancer areas, were then collected as formalin fixed paraffin-embedded (FFPE) samples. To confirm RCC subtypes and the Fuhrman nuclear grading $[10,11]$, in both cancer and adjacent non-cancer areas, hematoxylin and eosin (H\&E) stained slides were evaluated by a renal pathologist.

\section{Immunohistochemistry}

Immunohistochemistry was performed on both cancer and adjacent non-cancer renal tissues. Briefly, 4$\mu \mathrm{m}$-thick tissue sections were baked in a hot-air oven at $60^{\circ} \mathrm{C}$ for $1 \mathrm{~h}$, deparaffinized with xylene, and rehydrated with graded series of ethanol/water. Thereafter, the tissue slides were immersed in $0.3 \%$ 
Triton-X in PBS-T (PBS containing 0.3\% Tween-20; pH 7.4) for 40 min and then washed twice with PBS-T. Antigen retrieval was performed by using heat-induced epitope retrieval (HIER) protocol with a buffer containing $10 \mathrm{mM}$ Tris-Base, $1 \mathrm{mM}$ EDTA and $0.05 \%$ Tween-20 $(\mathrm{pH} 8.0)$ at $97^{\circ} \mathrm{C}$ for $30 \mathrm{~min}$. The tissue slides were then cooled down at $25^{\circ} \mathrm{C}$ for 20 min, washed twice with PBS-T, and incubated with $3 \%$ $\mathrm{H}_{2} \mathrm{O}_{2} /$ methanol for $10 \mathrm{~min}$. Non-specific bindings were blocked with $5 \% \mathrm{BSA}$ at $25^{\circ} \mathrm{C}$ for $30 \mathrm{~min}$. After washing three times with PBS-T, the tissue sections were incubated with rabbit polyclonal anti-ARID1A antibody (Sigma-Aldrich; St. Louis, MO) (diluted 1:400) at $25^{\circ} \mathrm{C}$ overnight and then with biotinylated goat anti-rabbit secondary antibody (Dako Corp.; Kyoto, Japan) (diluted $1: 200$ ) at $37^{\circ} \mathrm{C}$ for $2 \mathrm{~h}$. After washing with PBS-T three times, the sections were incubated with HRP-conjugated streptavidin (Dako Corp.) (diluted $1: 200$ ) at $25^{\circ} \mathrm{C}$ for $1 \mathrm{~h}$. After other three washes, the immunoreactive protein was colorized with DAB (3, 3'-diaminobenzidine) peroxidase substrate (Vector Laboratories; Burlingame, CA) for 4 min. The section stained without primary antibody served as a negative control. The stained sections were imaged under an Olympus BX50 light microscope (Olympus; Tokyo, Japan).

\section{Quantitative analysis of ARID1A protein expression}

Three randomized locales per slide were evaluated by the renal pathologist and the investigators in a double-blind fashion. Allred scoring and grading system, which is commonly used for quantitative analysis of estrogen/progesterone receptor in breast cancer [12], was employed for quantitative analysis of ARID1A protein expression in cancer and adjacent non-cancer renal tissues obtained from all RCC patients. Briefly, the Allred score considered both proportional score (PS) and intensity score (IS) that measured the proportion of the ARID1A-stained cells and the ARID1A intensity, respectively. The PS ranged from 0 (no ARID1A-stained cells) to 5 (>2/3 ARID1A-stained cells in each field), whereas the IS ranged from 0 (negative staining) to 3 (strong staining). Allred score was the summation of the PS and IS and was finally graded as negative (Allred score $=0-1$ ), weak (Allred score $=2-3$ ), moderate (Allred score = 4-5) and strong (Allred score $=6-8$ ). If there was any discrepancy of the PS and IS among the investigators, the scores were reassessed to find out the possible reasons for disagreement.

In addition, levels of ARID1A protein expression in various parts of the nephron, including glomerulus (Glom), proximal convoluted tubule (PCT), distal convoluted tubule (DCT) and collecting duct (CD), were evaluated in the adjacent non-cancer areas of cCRCC (grade I) versus cCRCC (grade II). The intensity of ARID1A (represented by DAB staining) was quantitated from at least 100 cells in each part of the nephron as aforementioned using Image $\mathrm{J}$ - Fiji analysis software (http://fiji.sc/Fiji).

\section{Association of ARID1A protein expression with clinicopathology and progression-free survival}

According to the Allred four grading system described above, ARID1A protein expression was categorized into "negative to weak expression" and "moderate to strong expression". Such categorization was then analyzed for its association with clinicopathology (using Pearson's chi-square and Fisher's exact tests) and cumulative progression-free survival (PFS) within five years after surgical removal (using Kaplan- 
Meier analysis and log-rank test). Finally, the univariate and multivariate analyses of PFS were analyzed by a clinical epidemiologist to evaluate the potential predictors of prognosis of patients with RCCs.

\section{Statistical analysis}

All quantitative data are presented as mean \pm SEM unless stated otherwise. Comparisons of the data between the paired samples were done by paired Student's t-test, whereas comparisons of the unpaired samples were done by unpaired Student's t-test or by Mann-Whitney test (when the data did not distribute normally). The association between ARID1A protein expression and clinicopathology was analyzed by Pearson's chi-square and Fisher's exact tests, whereas the association between ARID1A protein expression and progression-free survival was evaluated by Kaplan-Meier analysis and log-rank test. All statistical analyses were done through the SPSS software (version 16.0) (SPSS; Chicago, IL). The univariate and multivariate analyses of progressive-free survival were analyzed using a binary logistic regression model, followed by the Gaussian model, through the Stata SE version 16 for Windows (StataCorp LP, TX). Pvalues less than 0.05 were considered statistically significant.

\section{Results}

\section{ARID1A was one of the top-ten mutated genes most frequently found in kidney cancers and its mutations were found along its sequence}

Bioinformatics analysis through the Genomic Data Commons (GDC) database using all of The Cancer Genome Atlas (TCGA) projects related to kidney cancers, including TCGA-KIRC (kidney renal clear cell carcinoma), TCGA-KIRP (kidney renal papillary cell carcinoma), TCGA-KICH (kidney chromophobe), and TCGA-SARC (sarcoma) showed that ARID1A was one of the top-ten mutated genes most frequently found in kidney cancers (Fig. 1A). A total of 32 mutations related to kidney cancers were found in ARID1A gene, accounting for $4.03 \%$ of all the mutated genes detected in all the affected cases. In addition, these 32 mutations in ARID1A gene, including 14 frameshift (43.75\%), 14 missense mutations (43.75\%), and 4 stop gained (12.5\%) were found along its protein sequence (Fig. 1B). Interestingly, six of these mutations were found in the important domains, including ARID DNA-binding domain (PF01388) and SWI/SNF-like complex subunit BAF250/Osa (PF12031) domains (Fig. 1B). Moreover, patients with ARID1A mutations had significantly lower survival rate $(38 \% ; n=68)$ comparing to the non-mutated cases $(58 \% ; n=192)$ (Fig. 1C). We further analyzed the overall survival (OS) and disease specific survival (DSS) of patients with kidney renal clear cell carcinoma in TCGA and GEO databases using the OSkirc web tool. The results from the TCGA dataset showed that patients with low expression of ARID1A had significantly lower OS (HR: 0.66; 95\% Cl: 0.49-0.90; $p=0.0086$ ) and lower DSS (HR: 0.61; 95\% Cl: 0.41-0.90; $p=0.0125)$ when compared with those with high expression of ARID1A (Fig. 2A-B). Patients with low expression of $A R I D 1 A$ in the combined TCGA-GEO datasets also had significantly lower OS (HR: 0.71; 95\% Cl: 0.53-0.95; $p=$ 0.0195 ) and lower DSS (HR: 0.67; 95\% Cl: 0.47-0.96; $\mathrm{p}=0.0277$ ) when compared to those with high expression of ARID1A (Fig. 2C-D). 


\section{Histopathology and ARID1A protein expression in cancer vs. adjacent non-cancer areas}

The histopathology of cancer and adjacent non-cancer areas of the paired renal tissues collected from RCC patients, who were admitted at Sawanpracharak Hospital, Nakhon Sawan, Thailand for nephrectomy during 2013-2017, was examined with H\&E staining. The subtype and the Fuhrman nuclear grading were then evaluated. From a total of 26 RCC patients recruited, 17 had clear cell RCC (ccRCC) (six with Fuhrman nuclear grade I and 11 with Fuhrman nuclear grade II), six had chromophobe RCC (chRCC), two had papillary RCC (pRCC), and only one had sarcomatoid RCC (sRCC).

Immunohistochemistry was performed to evaluate ARID1A protein expression in these paired renal tissues (Fig. 3A). The data showed that ARID1A protein was strongly expressed in the nuclei of lymphocytes, fibroblasts, intraglomerular cells, and tubular epithelial cells of proximal convoluted tubules and distal convoluted tubules in the adjacent non-cancer areas, whereas lighter ARID1A stainings were observed in these cells in the cancer area (Fig. 3A). Quantitative analysis using Allred scoring and grading system revealed that ARID1A Allred score was significantly decreased in cancer areas of all RCC cases (Fig. 3B). Comparing in each subtype of RCC, ARID1A Allred score was markedly decreased in cancer area of ccRCC (grade I), RCC (grade II) and chRCC, whereas there was no significant difference observed in pRCC and SRCC (Fig. 3B). Similarly, Allred grading revealed that almost all the adjacent non-cancer area (25/26 cases) had moderate to strong ARID1A expression, whereas almost all the cancer area of ccRCC (grade I), ccRCC (grade II) and chRCC (21/23 cases) had negative to weak ARID1A expression (Fig. 3C). However, the three cases with pRCC and SRCC had moderate to strong ARID1A expression (Fig. 3C).

The progression-free survival (PFS) of patients with moderate to strong ARID1A vs. negative to weak ARID1A expression

The progression-free survival (PFS) of patients with moderate to strong ARID1A and negative to weak ARID1A expression was analyzed. All 9/9 RCC patients with moderate to strong ARID1A expression had progression-free survival rate of $100 \%$ (Fig. 3D). Log-rank test revealed a tendency of lower PFS in patients with negative to weak ARID1A expression $(n=17)$ as compared to those with moderate to strong ARID1A expression ( $n=9$ ) but the $p$ value did not reach the statistically significant threshold (Fig. 3D).

\section{Association of ARID1A protein expression and clinicopathological outcomes of patients}

Allred grading was then applied to compare clinicopathological outcomes of patients. There were 17 cases with "moderate to strong ARID1A expression" and nine cases with "negative to weak ARID1A expression". Their mean ages were comparable, whereas tumor mass diameter tended to be larger and progression-free duration tended to be shorter in patients with negative to weak ARID1A protein expression, although the statistically significant threshold was not reached (Table 1). Using Fisher's exact test, patients with negative to weak ARID1A expression had significantly greater number and percentage 
of ccRCC (grade II) and chRCC cases $(p=0.003)$, presence of comorbidity $(p=0.038)$, and low eGFR levels ( $p=0.008$ ) as compared to those with moderate to strong ARID1A expression (Table 2). However, their elderly (age $\geq 60$ years), gender, tumor laterality, TNM stage and AJCC stage made no significant differences between the two groups (Table 2). Univariate analysis revealed that negative expression of ARID1A in RCC tissues (29\% of all patients; $95 \%$ Cl: $0.07-0.52 ; p=0.009)$, high AJCC stage (II-IV) ( $26 \%$ of all patients; $95 \% \mathrm{Cl}: 0.06-0.47 ; p=0.011)$, and presentation with chronic kidney disease (31\% of all patients; $95 \% \mathrm{Cl}: 0.08-0.54 ; p=0.008$ ) were associated with a short PFS (Table 3 ). Multivariate analysis revealed that negative expression of ARID1A in RCC tissues and other parameters were not associated with a short PFS of patients (Table 3). Furthermore, ARID1A protein was undetectable in $3 / 11$ cases with ccRCC (grade II) and 2/6 chRCC cases, all of which had metastasis (to lung, brain, bone, and liver) 1-50 months after surgical removal.

Table 1

Clinical data of RCC patients (total $n=26$; moderate to strong ARID1A expression $=9$ cases; negative to weak ARID1A expression = 17 cases).

\begin{tabular}{|c|c|c|}
\hline Clinical data & Mean \pm SEM & $p$-value \\
\hline Age (years) & $61.00 \pm 1.94$ & \\
\hline Moderate to strong ARID1A expression & $61.00 \pm 3.91$ & $0.75^{a}$ \\
\hline Negative to weak ARID1A expression & $60.88 \pm 2.17$ & \\
\hline Tumor mass diameter (cm) & $7.91 \pm 0.61$ & \\
\hline Moderate to strong ARID1A expression & $6.23 \pm 1.17$ & $0.07^{b}$ \\
\hline Negative to weak ARID1A expression & $8.60 \pm 0.66$ & \\
\hline Progression-free duration (month) & $52.65 \pm 3.35$ & \\
\hline Moderate to strong ARID1A expression & $60.00 \pm 0.00$ & $0.24^{a}$ \\
\hline Negative to weak ARID1A expression & $48.82 \pm 4.91$ & \\
\hline a By Mann-Whitney U test; ${ }^{b}$ By unpaire & tudent's t-te & \\
\hline
\end{tabular}


Table 2

Association of ARID1A expression with clinicopathology of RCC patients (total $n=26$ ).

\begin{tabular}{|c|c|c|c|c|}
\hline \multirow[t]{3}{*}{ Parameters } & \multirow[t]{3}{*}{$\mathrm{n}(\%)$} & \multicolumn{2}{|l|}{ ARID1A expression } & \multirow[t]{3}{*}{$p$ value $^{\mathrm{C}}$} \\
\hline & & Moderate to strong & Negative to weak & \\
\hline & & n (\%) & $\mathrm{n}(\%)$ & \\
\hline \multicolumn{5}{|l|}{ Age (years) } \\
\hline$\geq 60$ years old & $13(50.0)$ & $3(11.5)$ & $10(38.5)$ & 0.411 \\
\hline$<60$ years old & $13(50.0)$ & $6(23.1)$ & 7 (26.9) & \\
\hline \multicolumn{5}{|l|}{ Gender } \\
\hline Male & $21(80.8)$ & $7(26.9)$ & $14(53.8)$ & 1.000 \\
\hline Female & $5(19.2)$ & $2(7.7)$ & $3(11.5)$ & \\
\hline \multicolumn{5}{|l|}{ Laterality } \\
\hline Right & $17(65.4)$ & $5(19.2)$ & $12(46.2)$ & 0.667 \\
\hline Left & $9(34.6)$ & $4(15.4)$ & 5 (19.2) & \\
\hline \multicolumn{5}{|c|}{ Pathological subtypes } \\
\hline ccRCC (Grade I) & $6(23.1)$ & $4(15.4)$ & $2(7.7)$ & 0.003 \\
\hline ccRCC (Grade II) & $11(42.3)$ & $2(7.7)$ & $9(34.6)$ & \\
\hline ChRCC & $6(23.1)$ & $0(0)$ & $6(23.1)$ & \\
\hline Others & $3(11.5)$ & $3(11.5)$ & $0(0)$ & \\
\hline \multicolumn{5}{|l|}{ T stage } \\
\hline T1 & $7(26.9)$ & $3(11.5)$ & $4(15.4)$ & 0.661 \\
\hline T2-4 & $19(73.1)$ & $6(23.1)$ & $13(50)$ & \\
\hline \multicolumn{5}{|l|}{$\mathrm{N}$ stage } \\
\hline NO & $22(84.6)$ & $7(26.9)$ & $15(57.7)$ & 0.591 \\
\hline N1 & $4(15.4)$ & $2(7.7)$ & $2(7.7)$ & \\
\hline \multicolumn{5}{|l|}{ M stage } \\
\hline MO & $24(92.3)$ & $9(34.6)$ & $15(57.7)$ & 0.529 \\
\hline M1 & $2(7.7)$ & $0(0)$ & $2(7.7)$ & \\
\hline \multicolumn{5}{|l|}{ AJCC stage } \\
\hline Stage I & $7(26.9)$ & $3(11.5)$ & $4(15.4)$ & 0.661 \\
\hline
\end{tabular}




\begin{tabular}{|c|c|c|c|c|}
\hline \multirow[t]{2}{*}{ Parameters } & \multirow[t]{2}{*}{$n(\%)$} & \multicolumn{2}{|l|}{ ARID1A expression } & \multirow[t]{2}{*}{$p$ value $^{c}$} \\
\hline & & $\begin{array}{l}\text { Moderate to strong } \\
\mathrm{n}(\%)\end{array}$ & $\begin{array}{l}\text { Negative to weak } \\
\mathrm{n}(\%)\end{array}$ & \\
\hline Stage II-IV & $19(73.1)$ & $6(23.1)$ & $13(50.0)$ & \\
\hline
\end{tabular}

Table 2

Association of ARID1A expression with clinicopathology of RCC patients (total $n=26$ )(Cont.).

\begin{tabular}{|lllll|}
\hline Parameters & $\mathbf{n}(\%)$ & \multicolumn{2}{l}{ ARID1A expression } & \multirow{2}{*}{ p value $^{\mathrm{C}}$} \\
\cline { 3 - 4 } & & $\begin{array}{l}\text { Moderate to strong } \\
\mathbf{n}(\%)\end{array}$ & $\begin{array}{l}\text { Negative to weak } \\
\mathbf{n}(\%)\end{array}$ & \\
\hline Comorbidity & & & & 0.038 \\
\hline Absence & $12(46.2)$ & $7(26.9)$ & $12(46.2)$ & \\
\hline Presence & $14(53.8)$ & $2(7.7)$ & & 0.008 \\
\hline eGFR & & & $3(11.5)$ & \\
\hline Normal & $10(38.5)$ & $7(26.9)$ & $7(26.9)$ & \\
\hline Mild to moderate low & $9(34.6)$ & $2(7.7)$ & $7(26.9)$ & \\
\hline Severe low & $7(26.9)$ & $0(0)$ & & \\
\hline
\end{tabular}


Table 3

Univariate and multivariate analyses of progressive-free survival of patients with RCCs using a binary logistic regression model and the Gaussian model.

\begin{tabular}{|c|c|c|c|c|c|c|}
\hline \multirow[t]{2}{*}{ Parameters } & \multicolumn{3}{|c|}{ Univariate analysis } & \multicolumn{3}{|c|}{ Multivariate analysis } \\
\hline & Coef. & $95 \% \mathrm{Cl}$ & $\mathrm{p}$ value & Coef. & $95 \% \mathrm{Cl}$ & $p$ value \\
\hline $\begin{array}{l}\text { ARID1A expression } \\
\text { Negative vs positive }\end{array}$ & 0.29 & $0.07-0.52$ & 0.009 & 0.10 & $-0.13-0.34$ & 0.369 \\
\hline $\begin{array}{l}\text { Age, years old } \\
\geq 60 \text { vs }<60\end{array}$ & 0.77 & $-0.23-0.38$ & 0.634 & 0.11 & $-0.00-0.02$ & 0.084 \\
\hline $\begin{array}{l}\text { Gender } \\
\text { Female vs male }\end{array}$ & 0.01 & $-0.38-0.41$ & 0.962 & 0.04 & $-0.33-0.42$ & 0.827 \\
\hline $\begin{array}{l}\text { Tumor laterality } \\
\text { Left vs right }\end{array}$ & 0.05 & $-0.28-0.38$ & 0.788 & 0.14 & $-0.19-0.48$ & 0.407 \\
\hline $\begin{array}{l}\text { Pathological grading } \\
\text { High grade vs low grade }\end{array}$ & 0.04 & $-0.08-0.16$ & 0.534 & -0.006 & $-0.09-0.08$ & 0.875 \\
\hline $\begin{array}{l}\text { AJCC stages } \\
\text { II-IV vs I }\end{array}$ & 0.26 & $0.06-0.47$ & 0.011 & 0.20 & $-0.04-0.45$ & 0.111 \\
\hline $\begin{array}{l}\text { Diabetes mellitus } \\
\text { Presence vs absence }\end{array}$ & 0.36 & $-0.16-0.88$ & 0.171 & 0.16 & $-0.26-0.59$ & 0.454 \\
\hline $\begin{array}{l}\text { Chronic kidney disease } \\
\text { Presence vs absence }\end{array}$ & 0.31 & $0.08-0.54$ & 0.008 & 0.06 & $-0.23-0.34$ & 0.701 \\
\hline
\end{tabular}

\section{Discussion}

The present study investigated $A R I D 1 A$ gene mutation in kidney cancers on the Genome Data Commons (GDC). The results showed that ARID1A mutation was one of the top-ten mutated genes most common found in kidney cancers together with PBRM1 (Polybromo 1), VHL (von Hippel-Lindau), TP53 (tumor suppressor 53), SETD2 (SET domain-containing protein 2), etc. Among these, mutations of PBRM1 and $V H L$ are the majority to cause cCRCC subtype $[13,14]$. Mutation of MET proto-oncogene and inactivation of SWI/SNF chromatin/histone modifiers are common in pRCC type I, whereas mutations of FH (fumarate hydratase), SETD2, and CDKN2A (cyclin dependent kinase inhibitor 2A) are more common in pRCC type II $[13,14]$. Chromophobe RCC is associated with mutations of PTEN (phosphatase and tensin homolog), TP53, and TERT (telomerase reverse transcriptase) mitochondrial genes [13, 14]. Consistent with our 
present study, a recent study by Ricketts and colleagues [15] has shown that mutations of SWI/SNF complex genes, including PBRM1, ARID1A and SMARCA4, were found notably in ccRCC (47.1\%), pRCC (53.0\%) and chRCC (14.9\%). It was thus plausible that $A R I D 1 A$ mutation might get involved in development and/or progression of RCC.

We further investigated mutation profiles of $A R I D 1 A$ gene in patients with kidney cancers. From a total of 32 mutations found, the majority came from frameshift (14/32) and missense (14/32), whereas only 4 had stop gained mutations. The mutation profiles occurred along the protein sequence of ARID1A, including the two conserved domains (ARID DNA-binding domain and SWI/SNF-like complex subunit BAF250/Osa domain on the C-terminus) of the Pfam structure. Human ARID1A, also known as BAF250a, comprises 2,285 amino acids with a molecular mass of approximately $250 \mathrm{kDa}$ and is a component of the SWI/SNF chromatin remodeling complex that regulates gene transcription [16]. The ARID DNAbinding domain (at residues 1019-1104) has been reported to play an important role in selective binding to AT-rich sites of DNA and assembly of polymorphic BRG-/BRM-associated factor (BAF) and polybromoassociated BAF (PBAF) complexes [17]. The C-terminal region domain of ARID1A (at residues 1976-2231) is essential for stimulating glucocorticoid receptor (GR)-mediated transcriptional activation [16]. Structural modeling has shown that ARID1A mediates GR interaction via the SWI/SNF complex that facilitates the transcriptional activation by GR [18]. Recurrent mutation and protein loss of ARID1A gene are most frequently found in ovarian clear cell carcinoma (mutation $=46-57 \%$ and protein loss $=41-59$ $\%$ ) and endometrial endometrioid carcinoma (mutation $=39-44 \%$ and protein loss $=19-34 \%$ ) [19-21]. Due to these mutations found in ARID1A, the structural and functional complexes of ARID1A protein and SWI/SNF would be expected to be affected in cancer patients.

Although three common predictive and prognostic markers, including VHL, VEGF (vascular endothelial growth factor) and CAIX (carbonic anhydrase IX) have been validated in RCC, their uses in clinical routine are still regarded as controversial [22]. Recently, P4HB (Prolyl 4-hydroxylase, beta polypeptide) [23] and five-proteins panel (APC; adenomatous polyposis coli, NOTCH1; neurogenic locus notch homolog protein 1, EYS; protein eyes shut homolog, filamin A and ARID1A) [24] have been proposed as novel, potential diagnostic and prognostic biomarkers for kidney renal clear cell carcinoma. In this study, we found that the patients with mutated ARID1A had a shorter overall survival than those without ARID1A mutations. The results from OSkirc web tool, which is a reliable and user-friendly analysis tool [9], also confirmed that the patients with low ARID1A expression had a significantly worse prognosis than those with high ARID1A expression. Consistently, several other studies have also reported that the patients with mutations or loss of $A R I D 1 A$ had a shorter disease-free survival and/or poorer overall outcome/survival [25-27]. Our results were in concordance with those reported previously indicating that $A R I D 1 A$ is an important tumor suppressor gene in RCC and may also serve as a prognostic biomarker in RCC patients.

Globally, most of the RCC cases are affected by three main RCC subtypes, including ccRCC (75\%), pRCC $(15 \%)$, and chRCC (5\%) [28]. Sarcomatoid differentiation is distinct in a high-grade and poorly differentiated components and is found in only $5 \%$ of RCC [10]. In this present study, we recruited a variety of RCC subtypes and grades, including ccRCC (grade I), ccRCC (grade II), chRCC, pRCC and sRCC 
from patients with age of $>45$ years following the recent classification system and histopathological descriptions [29]. To our knowledge, this is the first evidence demonstrating ARID1A protein expression in sRCC.

Our histopathological data showed that ARID1A is localized mainly in the nuclei of lymphocytes, fibroblasts, intraglomerular cells and renal tubular epithelial cells in the non-neoplastic areas. By contrast, nuclear ARID1A expression was diminished in the cancer cells, especially in ccRCC and chRCC, whereas no significant changes were observed in PRCC and SRCC. Recent studies have demonstrated that nuclear ARID1A protein was generally found in lymphocytes, fibroblasts and endothelial cells in various normal tissues [30-32]. Therefore, expression of ARID1A in these cells is frequently used as a positive control to compare with that of cancer cells [30-32]. However, the precise mechanisms of the defective ARID1A expression in these cancer cells have not been thoroughly investigated. A variety of extracellular matrices, stromal cells (such as fibroblasts, mesenchymal cells, pericytes, endothelial cells and lymphatic vascular networks) and immune cells (such as lymphocytes, natural killer cells and tumor-associated macrophages) participate in enhancing cancer cell survival, growth, invasion and metastasis [33, 34]. A recent evidence has suggested that cooperation of $A R I D 1 A$ and PIK3CA mutations enhanced proliferation of ovarian surface epithelial cells isolated from mice carrying mutant alleles by overproduction of interleukin-6 [35]. It is thus possible that ARID1A may help in the prevention of an inflammation-driven tumorigenesis in mouse ovarian clear-cell carcinoma [35]. Therefore, the interactions between the tumor microenvironment (defined as the interplay between the nascent cancer cells and their surrounding environment) and ARID1A expression in these cancer cells and their effects on cancer initiation, progression and metastasis should be further investigated.

In our present study, the decrease/loss of ARID1A expression was commonly found in the nuclei of the cancer cells, especially in ccRCC and chRCC, when compared with the paired adjacent non-cancer tissues. Such loss or decrease could be explained by mutations and molecular/epigenetic variations because the majority of the ARID1A mutations were the inactivation by nonsense and frameshift mutations throughout the gene that cloud lead to the loss of ARID1A protein expression [1, 21, 36]. Alternatively, inframe indel (insertion-deletion) mutations of $A R I D 1 A$ affecting the nuclear export signal could disturb the stability of ARID1A protein expression, like nonsense and frameshift mutations. Degradation of abnormal nuclear ARID1A is regulated by the nuclear ubiquitin-proteasome system [37]. Furthermore, epigenetic silencing might be also involved $[4,5]$. ARID1A gene promoter hypermethylation is recognized as the main etiologic factor for the decreasing ARID1A mRNA expression in many cancers. It has been shown that decreasing ARID1A mRNA expression is controlled by a repressive histone modification (H3K27Me3) in invasive breast cancers [4]. Promoter hypermethylation has been demonstrated to be related with low ARID1A mRNA expression in squamous cell carcinoma cell lines [5]. In kidney cancers, unmethylated CpG promoter of ARID1A gene has been reported [38]. However, the epigenetic analysis of ARID1A gene in renal cell carcinoma is still rarely conducted and further studies are needed to address the precise role of epigenetics in regulation of ARID1A expression. 
Currently, there is no clearly specific method for investigating ARID1A protein expression although some interpretation systems, such as the three staining grades [30] and Histo-score [39], had been used. In this study, we employed the Allred scoring and grading system to quantify ARID1A protein expression in the renal tissues. This strategy was also applied in a recent study evaluating ARID1A expression in breast cancer tissues [31]. The Allred scores of ARID1A protein expression in cancer tissues, particularly ccRCC (grade I), ccRCC (grade II) and chRCC, were significantly lower than in the paired non-cancer tissues, whereas those of pRCC and SRCC subtypes had no significant changes. Interestingly, a previous report has shown a similar results indicating that ARID1A protein expression was significantly decreased mostly in ccRCC [7]. In addition, the four-grade system allowed us to discriminate the RCC patients with negative to weak expression from those with moderate to strong expression. Previous studies have reported associations of the decrease/loss of ARID1A expression with clinical factors, such as the higher nuclear grade, larger tumor sizes, higher PTNM stage, and presence of metastatic of cancer in ccRCC patients [7, 8]. In this study, we showed that the decrease/loss of ARID1A expression was significantly associated with ccRCC (grade II) and chRCC subtypes, presence of comorbidity, and low eGFR levels. As a result, the RCC patients with negative to weak ARID1A expression tended to have lower progression-free survival, consistent with another study in cervical cancer demonstrating that the patients with ARID1A loss had significantly lower survival [40].

In this study, statistical analysis by a binary logistic regression model and the Gaussian model was also performed to assess the prognostic variable to a progressive-free survival of patients. Univariated analysis revealed that negative ARID1A expression, high AJCC stage (II-IV), and presence of chronic kidney disease were significantly associated with the progressive-free survival, but negative ARID1A expression was not an independent prognostic factor for patients with RCCs in multivariate analysis. However, due to the small sample size, the prognostic significance of ARID1A expression in RCCs requires clarification in further studies, using the Cox proportional hazard model with adequate sample size.

In summary, we have shown herein that $A R I D 1 A$ was one among the most common mutated genes found in kidney cancers. Immunohistochemistry demonstrated that ARID1A protein expression was markedly decreased in the RCC cancer tissues (particularly ccRCC and chRCC) as compared to the adjacent noncancer area. Additionally, level of ARID1A protein expression in the adjacent non-cancer renal tissues was significantly lower in ccRCC (grade II) than in CcRCC (grade I). Moreover, all of the RCC cases with negative ARID1A protein expression (3/11 cases with ccRCC (grade II) and 2/6 chRCC cases) had metastasis 1-50 months after the surgical removal. Finally, progression-free survival tended to be shorter in RCC patients with negative to weak ARID1A expression. Taken together, these data implicate that the defect/loss of ARID1A expression is associated with poor prognosis and metastasis of RCC and thus may serve as the prognostic marker of RCC, particularly $\mathrm{cCRCC}$ and chRCC subtypes.

\section{Declarations}

\section{Conflict of interest statement}


Authors declare that there is no conflict of interest in the research.

\section{Acknowledgements}

We would like to thank all members at Department of Anatomical Pathology, Sawanpracharak Hospital for their help and support in sample collection and preparation as well as patients' data acquisition. We are also grateful to Dr. Chitlada Utaipiboon for their suggestion in statistics and to Dr. Roger Callahan, Mea Fah Luang University for his English editing service. VT was supported by Mahidol University research grant and the Thailand Research Fund (IRN60W0004 and IRG5980006). NS was supported by the Thailand Science Research and Innovation (TSRl; grant number R2564B002) and Faculty of Medical Science, Naresuan University, Phitsanulok, Thailand.

\section{Author contributions}

KS, RS, VT and NS designed research; KS, RS, and YS collected sample and performed experiments; KS, RS, PT, SA, VT and NS analyzed data; KS, SA, VT and NS wrote the manuscript; All authors reviewed and approved the manuscript; NS final approved and submitted manuscript

\section{Ethical approval}

The study protocol involving human subjects was approved by the Human Ethic Review Board of Sawanpracharak Hospital (approval no. 47/2560) and by Naresuan University Ethical Committee for Human Research (NU-IRB) (approval no. 0489/61; COE no. 115/2018) and was conducted in accordance with the Declaration of Helsinki Principles.

\section{References}

1. Wu JN, Roberts CW. ARID1A mutations in cancer: another epigenetic tumor suppressor? Cancer Discovery. 2013;3(1):35-43. DOI:10.1158/2159-8290.cd-12-0361.

2. Yan HB, Wang XF, Zhang Q, Tang ZQ, Jiang YH, Fan HZ, et al. Reduced expression of the chromatin remodeling gene ARID1A enhances gastric cancer cell migration and invasion via downregulation of E-cadherin transcription. Carcinogenesis. 2014;35(4):867-76. DOI:10.1093/carcin/bgt398.

3. Mathur R, Alver BH, San Roman AK, Wilson BG, Wang X, Agoston AT, et al. ARID1A loss impairs enhancer-mediated gene regulation and drives colon cancer in mice. Nature Genetics 2017;49(2):296302. DOI: $10.1038 / \mathrm{ng} .3744$.

4. Zhang X, Sun Q, Shan M, Niu M, Liu T, Xia B, et al. Promoter Hypermethylation of ARID1A Gene Is Responsible for Its Low mRNA Expression in Many Invasive Breast Cancers. PLOS ONE. 2013;8(1):e53931. DOI:10.1371/journal.pone.0053931.

5. Luo Q, Wu X, Chang W, Zhao P, Zhu X, Chen H, et al. ARID1A Hypermethylation Disrupts Transcriptional Homeostasis to Promote Squamous Cell Carcinoma Progression. Cancer research. 2020;80(3):406. DOI:10.1158/0008-5472.CAN-18-2446. 
6. Wang X, Nagl NG, Jr., Flowers S, Zweitzig D, Dallas PB, Moran E. Expression of p270 (ARID1A), a component of human SWI/SNF complexes, in human tumors. International Journal of Cancer. 2004;112(4):636. DOI:10.1002/ijc.20450.

7. Lichner Z, Scorilas A, White NM, Girgis AH, Rotstein L, Wiegand KC, et al. The chromatin remodeling gene ARID1A is a new prognostic marker in clear cell renal cell carcinoma. American Journal of Pathology. 2013;182(4):1163-70. DOI:10.1016/j.ajpath.2013.01.007.

8. Park JH, Lee C, Suh JH, Chae JY, Kim HW, Moon KC. Decreased ARID1A expression correlates with poor prognosis of clear cell renal cell carcinoma. Human Pathology. 2015;46(3):454-60. DOl:https://doi.org/10.1016/j.humpath.2014.12.002.

9. Xie L, Wang Q, Dang Y, Ge L, Sun X, Li N, et al. OSkirc: a web tool for identifying prognostic biomarkers in kidney renal clear cell carcinoma. Future Oncology. 2019;15(27):3103-10. DOI:10.2217/fon-2019-0296.

10. Corti B, Zucchini N, Fabbrizio B, Martorana G, Schiavina R, Grigioni ADE, et al. Pathology and Molecular Pathogenesis of Renal Cell Carcinoma. European Urology Supplements. 2006;5(8):573-9. DOI:10.1016/j.eursup.2006.03.005.

11. Fuhrman SA, Lasky LC, Limas C. Prognostic significance of morphologic parameters in renal cell carcinoma. American Journal of Pathology. 1982;6(7):655-63.

12. Qureshi A, Pervez S. Allred scoring for ER reporting and it's impact in clearly distinguishing ER negative from ER positive breast cancers. The Journal of the Pakistan Medical Association. 2010;60(5):350-3.

13. Cairns P. Renal Cell Carcinoma. Cancer Biomarkers. 2011;9(1-6):461-73. DOI:10.3233/CBM-20110176.

14. Lindgren D, Sjolund J, Axelson H. Tracing Renal Cell Carcinomas back to the Nephron. Trends Cancer. 2018;4(7):472-84. DOI:10.1016/j.trecan.2018.05.003.

15. Ricketts CJ, De Cubas AA, Fan H, Smith CC, Lang M, Reznik E, et al. The Cancer Genome Atlas Comprehensive Molecular Characterization of Renal Cell Carcinoma. Cell Reports. 2018;23(1):31326.e5. DOI:10.1016/j.celrep.2018.03.075.

16. Nie Z, Xue Y, Yang D, Zhou S, Deroo BJ, Archer TK, et al. A specificity and targeting subunit of a human SWI/SNF family-related chromatin-remodeling complex. Molecular and Cellular Biology. 2000;20(23):8879-88.

17. Sandhya S, Maulik A, Giri M, Singh M. Domain architecture of BAF250a reveals the ARID and ARMrepeat domains with implication in function and assembly of the BAF remodeling complex. PLOS ONE. 2018;13(10):e0205267. DOI:10.1371/journal.pone.0205267.

18. Muratcioglu S, Presman Diego M, Pooley John R, Grøntved L, Hager Gordon L, Nussinov R, et al. Structural Modeling of GR Interactions with the SWI/SNF Chromatin Remodeling Complex and C/EBP. Biophysical Journal. 2015;109(6):1227-39. DOl:https://doi.org/10.1016/j.bpj.2015.06.044.

19. Zhang Z-m, Xiao S, Sun G-y, Liu Y-p, Zhang F-h, Yang H-f, et al. The Clinicopathologic Significance of the Loss of BAF250a (ARID1A) Expression in Endometrial Carcinoma. International Journal of 
Gynecological Cancer. 2014;24(3):534-40. DOI:10.1097/igc.0000000000000092.

20. Wiegand KC, Shah SP, Al-Agha OM, Zhao Y, Tse K, Zeng T, et al. ARID1A mutations in endometriosisassociated ovarian carcinomas. New England Journal of Medicine. 2010;363(16):1532-43.

DOI:10.1056/NEJMoa1008433.

21. Wu R-C, Wang T-L, Shih I-M. The emerging roles of ARID1A in tumor suppression. Cancer Biology \& Therapy. 2014;15(6):655-64. DOI:10.4161/cbt.28411.

22. Rabjerg M. Identification and validation of novel prognostic markers in Renal Cell Carcinoma. Danish Medical Journal. 2017;64(10).

23. Xie L, Li H, Zhang L, Ma X, Dang Y, Guo J, et al. Autophagy-related gene P4HB: a novel diagnosis and prognosis marker for kidney renal clear cell carcinoma. Aging. 2020;12(2):1828-42. DOI:10.18632/aging.102715.

24. Kim J, Park J-Y, Shin S-J, Lim BJ, Go H. Neo-Fs Index: A Novel Immunohistochemical Biomarker Panel Predicts Survival and Response to Anti-Angiogenetic Agents in Clear Cell Renal Cell Carcinoma. Cancers. 2021;13(6). DOI:10.3390/cancers13061199.

25. Katagiri A, Nakayama K, Rahman MT, Rahman M, Katagiri H, Nakayama N, et al. Loss of ARID1A expression is related to shorter progression-free survival and chemoresistance in ovarian clear cell carcinoma. Modern Pathology. 2011;25:282. DOI:10.1038/modpathol.2011.161.

26. He F, Li J, Xu J, Zhang S, Xu Y, Zhao W, et al. Decreased expression of ARID1A associates with poor prognosis and promotes metastases of hepatocellular carcinoma. Journal of Experimental \& Clinical Cancer Research. 2015;34:47. DOI:10.1186/s13046-015-0164-3.

27. Yang L, Wei S, Zhao R, Wu Y, Qiu H, Xiong H. Loss of ARID1A expression predicts poor survival prognosis in gastric cancer: a systematic meta-analysis from 14 studies. Scientific Reports. 2016;6:28919-. DOI:10.1038/srep28919.

28. Banumathy G, Cairns P. Signaling pathways in renal cell carcinoma. Cancer Biology \& Therapy. 2010;10(7):658-64. DOI:10.4161/cbt.10.7.13247.

29. Zhou M, He H. Pathology of Renal Cell Carcinoma. In: Campbell SC, Rini BI, editors. Renal Cell Carcinoma: Clinical Management, Current Clinical Urology. New York: Springer Science+Business Media; 23-41. 2003. DOI:10.1007/978-1-62703-062-5_2.

30. Agaimy A, Cheng L, Egevad L, Feyerabend B, Hes O, Keck B, et al. Rhabdoid and Undifferentiated Phenotype in Renal Cell Carcinoma: Analysis of 32 Cases Indicating a Distinctive Common Pathway of Dedifferentiation Frequently Associated With SWI/SNF Complex Deficiency. American Journal of Surgical Pathology. 2017;41(2):253-62. DOI:10.1097/pas.0000000000000787.

31. Takao C, Morikawa A, Ohkubo H, Kito Y, Saigo C, Sakuratani T, et al. Downregulation of ARID1A, a component of the SWI/SNF chromatin remodeling complex, in breast cancer. Journal of Cancer. 2017;8(1):1-8. DOI:10.7150/jca.16602.

32. Zhang L, Wang C, Yu S, Jia C, Yan J, Lu Z, et al. Loss of ARID1A Expression Correlates With Tumor Differentiation and Tumor Progression Stage in Pancreatic Ductal Adenocarcinoma. Technology in Cancer Research \& Treatment. 2018;17:1533034618754475. DOI:10.1177/1533034618754475. 
33. Chen F, Zhuang X, Lin L, Yu P, Wang Y, Shi Y, et al. New horizons in tumor microenvironment biology: challenges and opportunities. BMC medicine. 2015;13:45-. DOI:10.1186/s12916-015-0278-7.

34. Wang M, Zhao J, Zhang L, Wei F, Lian Y, Wu Y, et al. Role of tumor microenvironment in tumorigenesis. Journal of Cancer. 2017;8(5):761-73. DOI:10.7150/jca.17648.

35. Chandler RL, Damrauer JS, Raab JR, Schisler JC, Wilkerson MD, Didion JP, et al. Coexistent ARID1APIK3CA mutations promote ovarian clear-cell tumorigenesis through pro-tumorigenic inflammatory cytokine signalling. Nature communications. 2015;6:6118-. DOI:10.1038/ncomms7118.

36. Guan B, Mao TL, Panuganti PK, Kuhn E, Kurman RJ, Maeda D, et al. Mutation and loss of expression of ARID1A in uterine low-grade endometrioid carcinoma. American Journal of Surgical Pathology. 2011;35(5):625-32. DOI:10.1097/PAS.0b013e318212782a.

37. Guan $B$, Gao M, Wu CH, Wang TL, Shih le M. Functional analysis of in-frame indel ARID1A mutations reveals new regulatory mechanisms of its tumor suppressor functions. Neoplasia. 2012;14(10):98693.

38. Ibragimova I, Maradeo ME, Dulaimi E, Cairns P. Aberrant promoter hypermethylation of PBRM1, BAP1, SETD2, KDM6A and other chromatin-modifying genes is absent or rare in clear cell RCC. Epigenetics. 2013;8(5):486-93. DOI:10.4161/epi.24552.

39. Numata M, Morinaga S, Watanabe T, Tamagawa H, Yamamoto N, Shiozawa M, et al. The clinical significance of SWI/SNF complex in pancreatic cancer. International Journal of Oncology. 2013;42(2):403-10. DOI:10.3892/ijo.2012.1723.

40. Cho H, Kim JS-Y, Chung H, Perry C, Lee H, Kim J-H. Loss of ARID1A/BAF250a expression is linked to tumor progression and adverse prognosis in cervical cancer. Human Pathology. 2013;44(7):1365-74. DOl:https://doi.org/10.1016/j.humpath.2012.11.007.

\section{Figures}


A

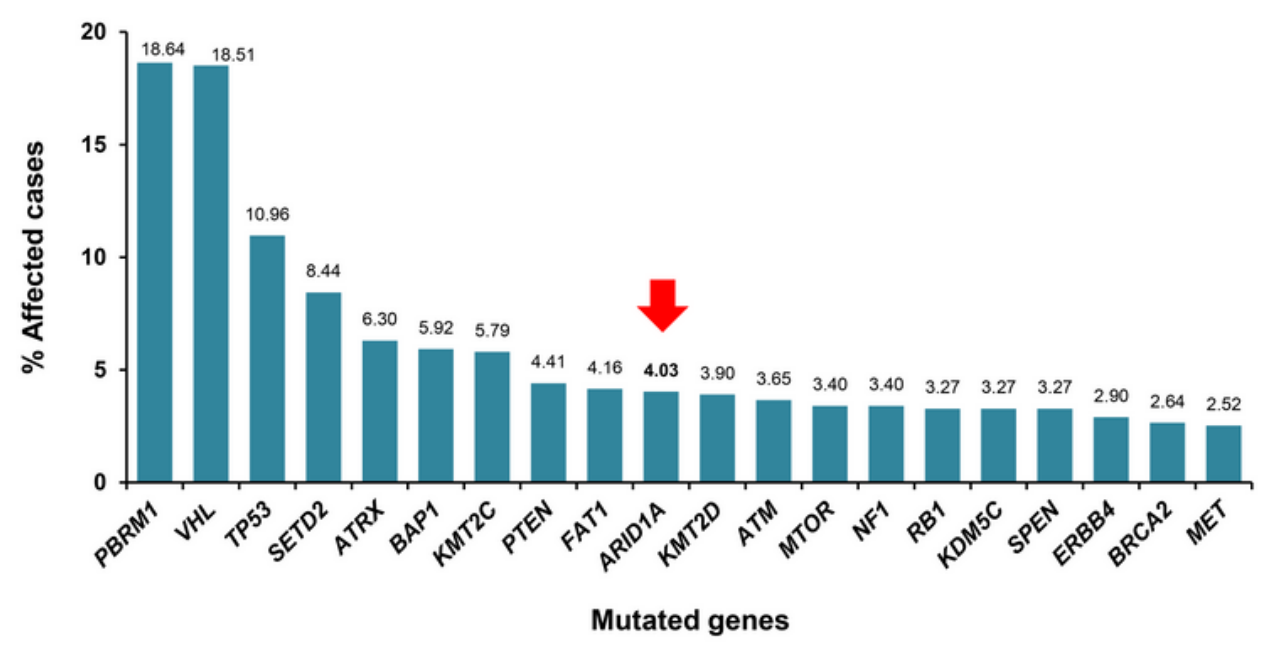

B

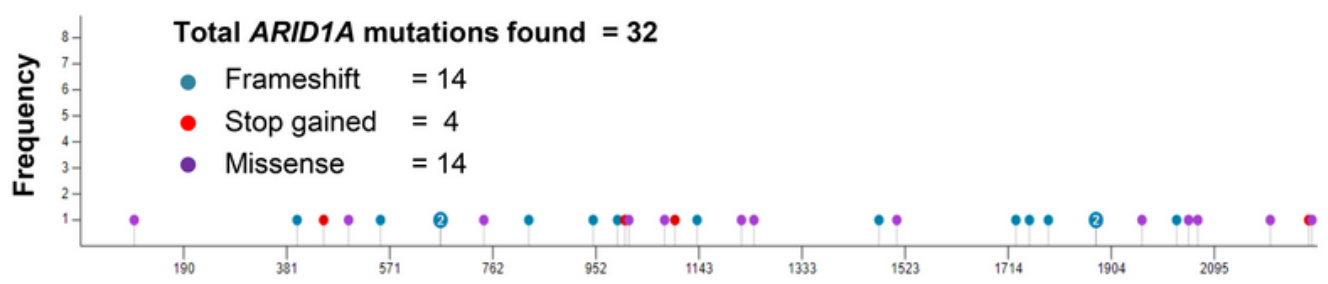

pfam

PF01388

PF12031

C $\mathrm{S}_{1}(\mathrm{n}=192):$ ARID1A non-mutated cases
$\mathrm{S}_{2}(\mathrm{n}=68):$ ARID1A mutated cases

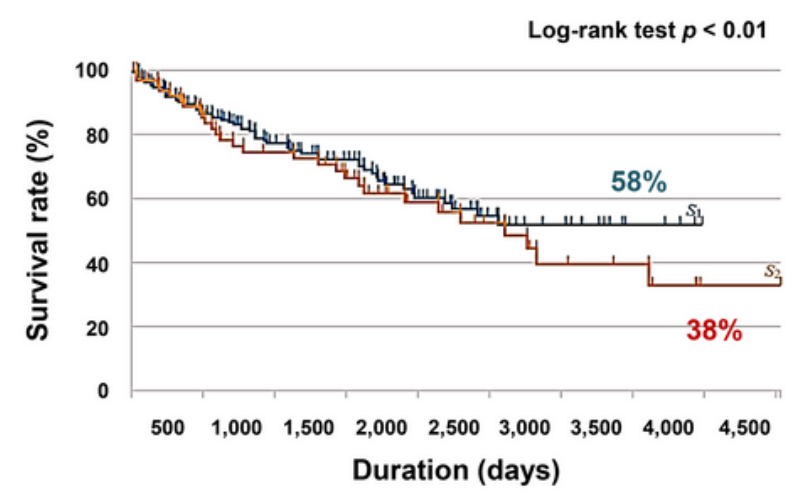

\section{Figure 1}

Gene mutations in kidney cancers. The mutated genes in kidney cancers were analyzed using the Genomic Data Commons (GDC) database via all of The Cancer Genome Atlas (TCGA) projects related to kidney cancers, including TCGA-KIRC (kidney renal clear cell carcinoma), TCGA-KIRP (kidney renal papillary cell carcinoma), TCGA-KICH (kidney chromophobe), and TCGA-SARC (sarcoma). (A): The top-20 mutated genes in kidney cancers found. The arrow indicates that ARID1A was one of the top-10 mutated 
genes found. (B): Frequency and mapping of frameshift, stop gained, and missense mutations of ARID1A gene along its protein sequence and ARID DNA-binding domain (PF01388) and SWI/SNF-like complex subunit BAF250/Osa (PF12031) domains. (C): Survival rate of patients with the mutated ARID1A versus that of the cases without ARID1A mutation.
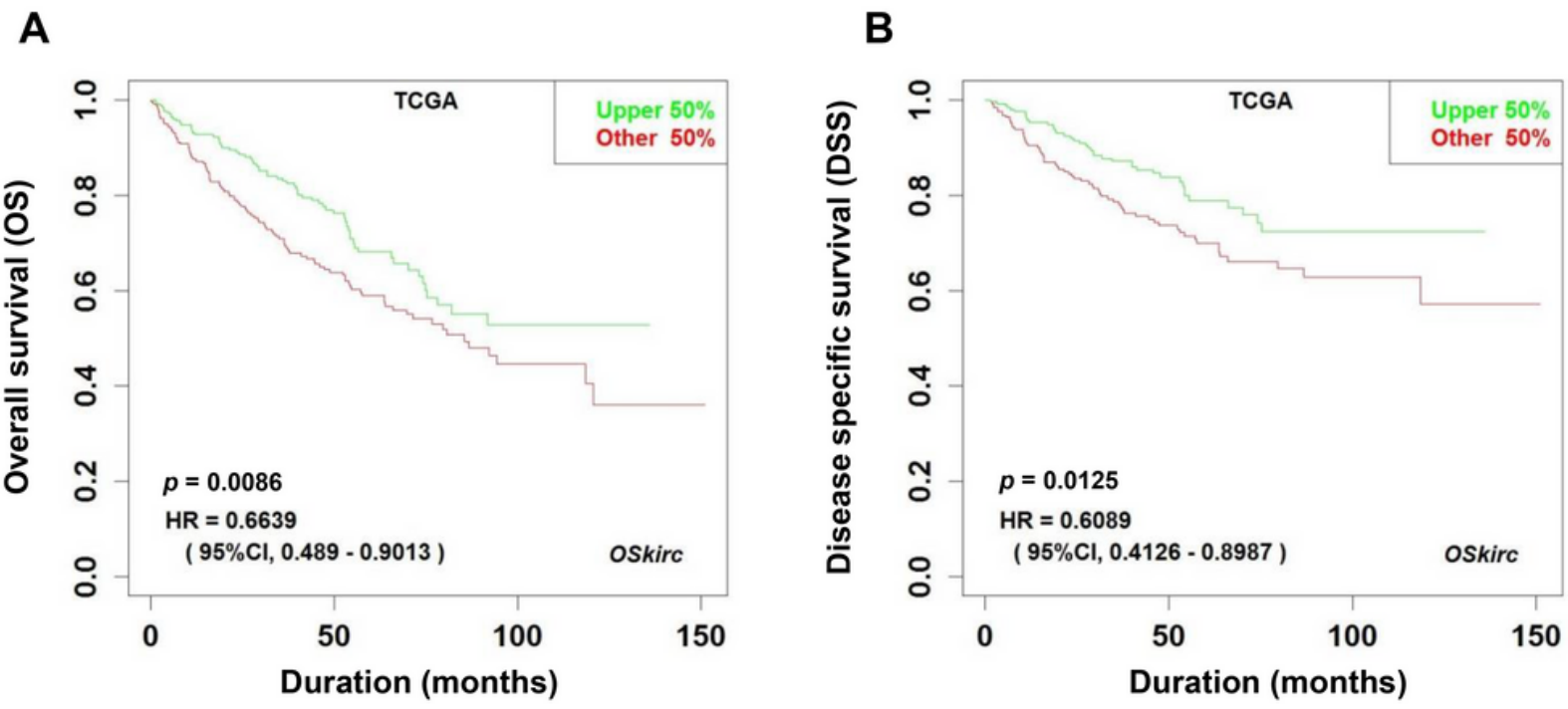

C

D
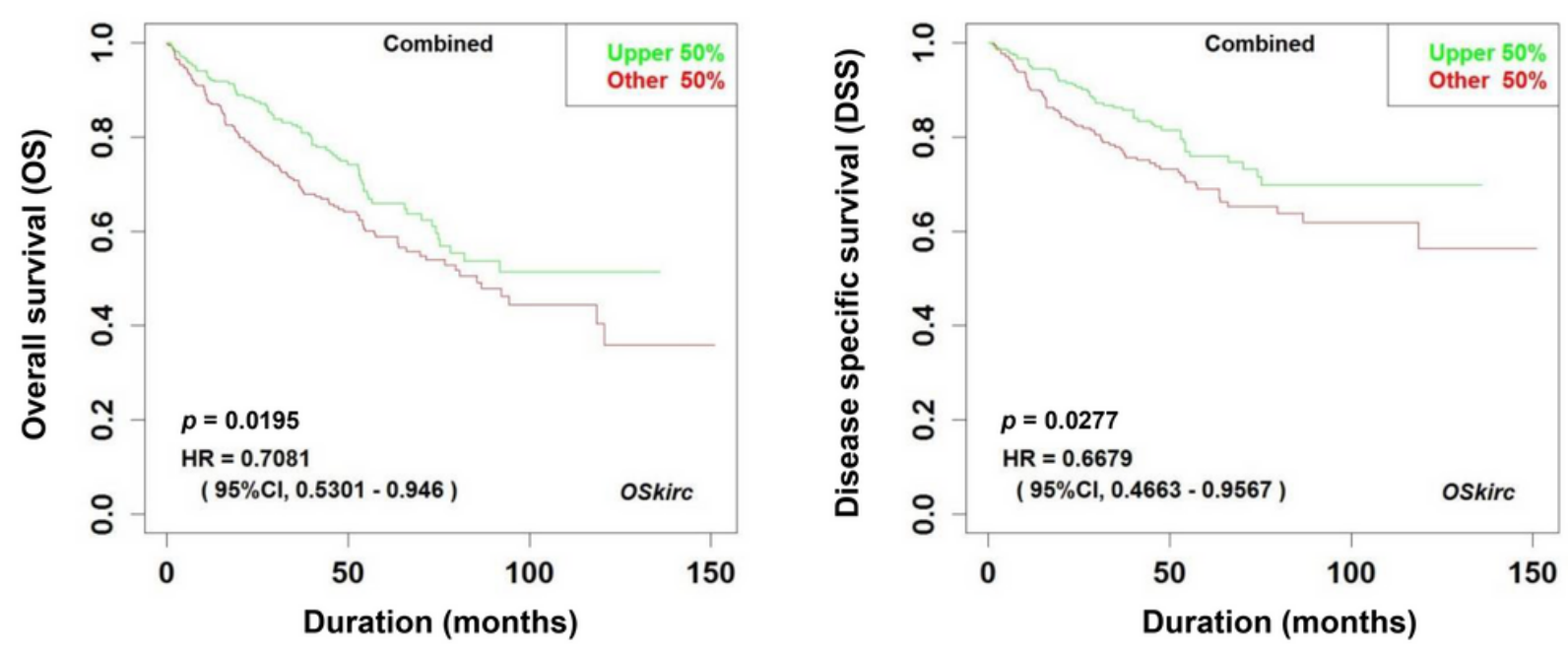

\section{Figure 2}

The overall survival (OS) and disease specific survival (DSS) in patients with kidney renal cell carcinoma. The OS and DSS of patients with kidney renal clear cell carcinoma were analyzed using the Online consensus Survival analysis for KIRC (OSkirc) web tool. (A-B): OS and DSS of patients in TCGA dataset. (C-D): The OS and DSS of patients in the combined TCGA-GEO datasets. The OS and DSS of patients 
with low expression of ARID1A (red) versus that of the cases with high expression of ARID1A (green). Abbreviations used: $\mathrm{HR}$, hazard ratio; $95 \% \mathrm{Cl}, 95 \%$ confidence interval.

A

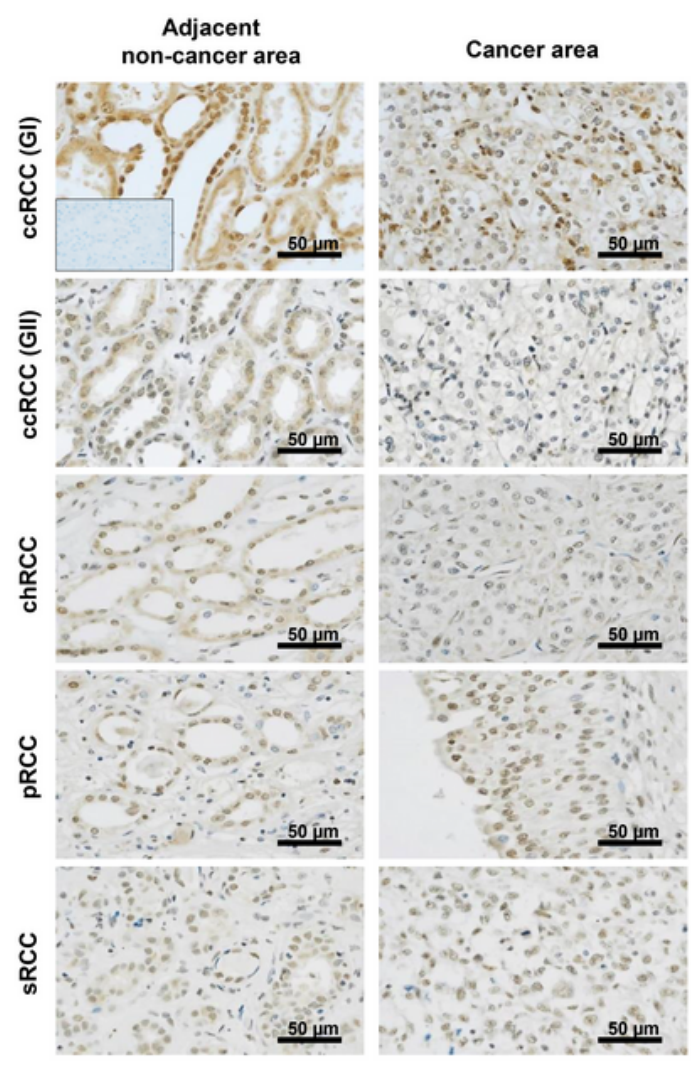

B
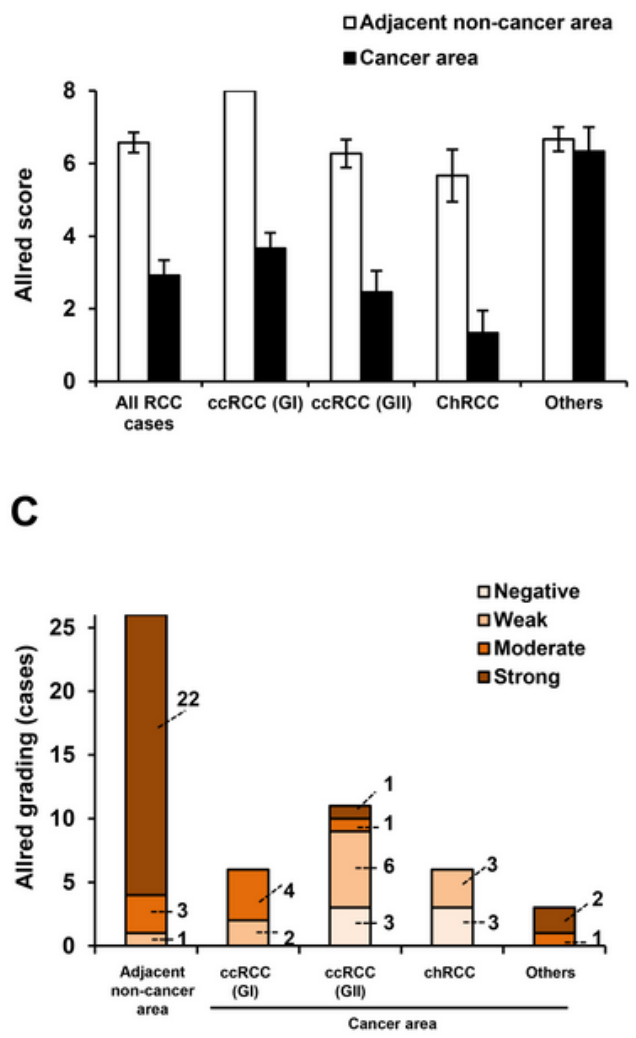

D

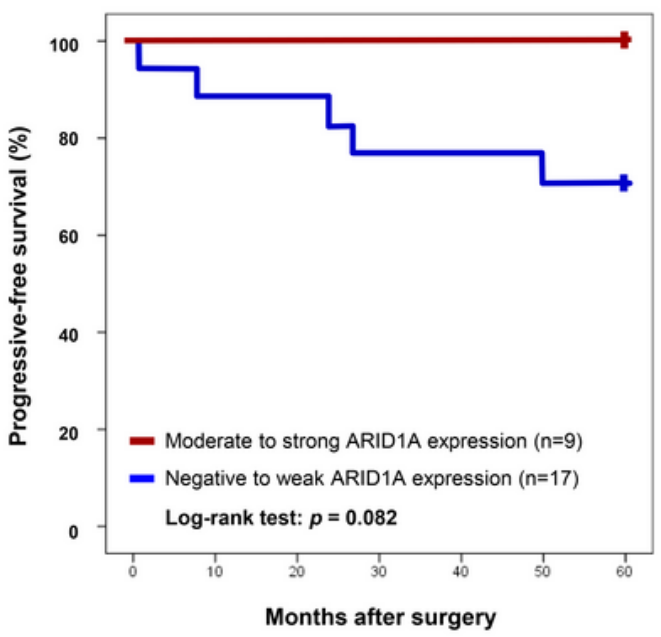

Figure 3

ARID1A protein expression in renal tissues from RCC patients and progressive free survival of patients. (A): ARID1A immunohistochemistry of the adjacent non-cancer area in ccRCC grade I (GI), ccRCC grade II (GII), chRCC, pRCC and SRCC, compared with the cancer area, respectively. ARID1A staining is observed in 
brown. An inset in panel A shows the negative control for ARID1A staining. Original magnification power of 400x for all panels. (B): Allred score of the adjacent non-cancer area versus cancer area in ccRCC grade I (GI), ccRCC grade II (GII), chRCC, and others (including pRCC and SRCC). The data are presented as mean \pm SEM. (C): Distribution and number of the cases with different Allred grades in the adjacent non-cancer and cancer areas. (D): The progression-free survival was analyzed in all RCC cases $(n=26)$ using log-rank test. 